\title{
concepto del coeficiente de seguridad en las obras de impermeabilización*
}

Carlos Safránez, Dr. Ingeniero

sinopsis

Para asegurar la eficacia de una impermeabilización hay que contar con un coeficiente de seguridad adecuado, pero observamos que su concepto resulta poco definido, precisamente en las obras de impermeabilización.

Al no ser factible aún expresar su coeficiente de seguridad en términos numéricos, por medio de un cálculo, a pesar de los muy notables progresos realizados últimamente en este sentido para lograrlo, tenemos que conformarnos, para las obras de impermeabilización, con un coeficiente de seguridad comparativo.
Se exponen los distintos factores que condicionan la eficacia de una impermeabilización, que hay que tener en cuenta y para la fijación de un coeficiente de seguridad adecuado.

Además, se debe tener presente que ciertas circuns tancias, como el emplazamiento de la obra, las condiciones sociales de los usuarios de la obra impermeabilizada, etc., pueden hacer aconsejable elegir una solución más segura que la mínima admisible por la Norma.

Finalmente, se indica un procedimiento para compa rar, por medio de un cálculo, el coeficiente de resismasilla asfáltica de sellado, de distintos tipos de jun tes de contracción.

\section{1. introducción}

Se observa en España, en los últimos años, un esfuerzo serio para mejorar la calidad en la Construcción, lo que lleva consigo también poder contar con un coeficiente de seguridad adecuado en las obras de impermeabilización.

El coeficiente de seguridad, uno de los conceptos fundamentales de la técnica, está definido, generalmente, para su empleo adecuado, o sea, para ofrecer el margen de seguridad que se pretende conseguir sin sobrepasar las naturales limitaciones económicas.

En cambio, cuando se trata de obras de impermeabilización y según lo demuestra la experiencia, el concepto del coeficiente de seguridad carece de la claridad precisa, y se observa con cierta frecuencia que las soluciones previstas no tienen la seguridad necesaria, lo que trae consigo la aparición de defectos, algunas veces de consecuencias muy graves.

Por otro lado, ante la sensación de inseguridad, al no pisar terreno firme y para no depender del factor suerte, se tiende en algunos casos a exagerar la seguridad de la impermeabilización, lo que produce gastos no del todo justificados.

La falta de claridad sobre el coeficiente de seguridad en obras de impermeabilización se debe, sobre todo, al gran número de factores que influyen sobre la eficacia de la impermeabilización, los c!ıales vamos a exponer en el transcurso de este trabajo.

\footnotetext{
* Conferencia pronunciada en el I Congreso Internacional de la Impermeabilización de Barcelona, mayo 80.
} 


\section{bibliografía}

1. Braun-Metelmann-Thun-Vordermeier. "Die Berechnung bituminöser Bauwerksabdichtungen». Arbeitsgemein schaft der Bitumen-Industrie e.V., 1976.

2. Dr. E. Braun «Portée des méthodes de calcul des étenchéités bitumineuses, sur le plan de la pratique». Journées d'Etudes A.I.E. (Association Internationale de I'Etenchéitél Venecia, 12 y 13 de mayo de 1977.

3. J. Y. Meynard "Théorie du travail élastique des revêtements d'étenchéité dans un système de toiture».

4. Document Technique Unifié, octobre 1975. N. ${ }^{\circ}$ 43. Travaux d'Etenchéité des Toitures Terrasses et des Toitures inclinées. Cahier des Charges.

5. C. Safránez «Impermeabilización de Cubiertas». Editoria Paraninfo, Madrid, 1973

6. C. Safránez "Consideraciones sobre el Hormigón Imper meable y el Aspecto de su Garantia». Informes de la Construcción. N. ${ }^{\circ}$ 233. Año XXIV Instituto Eduardo Torroja. Madrid.

7. Pierre Geisen «Definition des qualités des supports en fonction de leurs réactions sur les revêtements d'éten- chéité». Journées d'Etudes A.I.E. Venecia, 12 y 13 de mayo de 1977.

8. M. Stam "Développement de l'application des matériaux bitumineux pour l'étenchéisation des toitures, et des systémes sur différents matériaux d'isolation thermiques et autres». Journées d'Etudes A.I.E. Venecia, 12 y 13 de mayo de 1977.

9. L. Logeais "Les Toitures-Terrasses. Pathologie du Gros Oeuvre». Revue Générale de l'Etenchéité et de l'Isolation N. ${ }^{\circ} 95,96$ y 97, año 1974.

10. C. Safránez "La Garantia en las Obras de Impermeabilización». Temas de Arquitectura y Urbanismo. N. ${ }^{\circ} 126$. Madrid.

11. Normas Tecnológicas de la Edificación. NTE-QAT/1973 "Cubiertas: Azoteas transitables». NTE-QAN/1973 «Cubiertas: Azoteas no transitables"

12. Dr. E. Braun Journées d'Etudes A.I.E. Venecia, 12 y 13 de mayo de 1977. «Discussions». Págs. 17-23.

13. C. Safránez "Juntas de Contracción en Canales y Depósitos de Agua». Editorial Paraninfo, Madrid, 1977

\section{résumé}

CONCEPT DU COEFFICIENT DE SE CURITE DANS LES TRAVAUX D'IM PERMEABILISATION

Carlos Safránez, Dr. ingénieur

Pour assurer l'efficacité d'une imperméabilisation, il faut avoir un coefficient de sécurite approprié, mais nous constatons que son concept est peu défini justement dans les travaux d'imperméablisation.

Comme il n'est pas encore facile à exprimer. par calcul, leur coefficient de sécurité en termes numériques, malgré les progrès très importants récemment faits dans ce sens. nous devons nous contenter, pour les travaux d'imperméabilisation, d'un coefficient de sécurité comparatif.

On expose les différents facteurs condition nant l'efficacité d'une imperméabilisation, dont il faut tenir compte pour la fixation d'un cofficient de sécurité approprié.

En plus, il ne faut pas oublier le fait que certaines circonstances, telles que l'emplacement de l'ouvrage, les conditions sociales des usagers de l'ouvrage imperméablisé, etc. peuvent conseiller de choisir une solution peuvent conseiller de choisir une solution
plus sûre que la solution minimale admissible par la norme.

Finalement, on indique un procédé pour com parer, par calcul, le coefficient de résistance parer, par calcul, le coefficient de résistance d'asphalte de scellement, de différents types de joints de contraction.

\section{summary \\ SAFETY COEFFICIENT CONCEPT IN WATERPROOFING WORKS \\ Carlos Safránez, Dr. Engineer}

To assure the efficiency of waterproofing, a suitable safety coefficient should be counted on, but we find this concept is not clearly defined precisely in the waterproofing works.

Since it is not yet feasible to express the safety coefficient in numerical terms, using a calculation method, in spite of the most notable progress recently made in this respect to achieve this, we have to conform with a to achieve this, we have to conform with a
comparative safety coefficient, for waterproofing works.

The various factors that condition the efficiency of a waterproofing are described, and which must be considered when fixing a suitable safety coefficient.

It should moreover be remembered that cer tain circunstamces such as the location of th works, the social conditions of the wate proofed work users, etc., may make it advisable to choose a safer solution than the minimum accepted by the Standard.

Finally a procedure is given, through calcula tion, to compare the ageing and shedding re sistance coefficient of the asphalt sealer putty. of different kinds of construction joints.

\section{zusammenfassung}

BEGRIFF DES SICHERHEITSKOEFFI ZIENTEN FUER ABDICHTUNGSAR BEITEN

Carlos Safránez, Dr. Ingenieur

Zur Gewährleistung einer Abdichtung bedar es eines geeigneten Sicherheitskoeffizienten, doch stellen wir fest, dass dessen Begrif gerade für Abdichtungsarbeiten wenig definiert ist.

Da es trotz der beachtlichen Fortschritte, die in letzter Zeit in dieser Hinsicht erzielt wurden, nicht möglich ist, den Sicherheitskoeffizienten zahlenmässig zu berechnen, müssen wir uns für Abdichtungsarbeiten mit einem Vergleichs sicherheitskoeffizienten begnügen.

Es werden hier die verschiedenen Faktoren dargelegt, die die Wirksamkeit der Abdichtung bedingen und die für die Festlegung eines geeigneten Sicherheitskoeffizienten zu be rücksichtigen sind.

Es ist ferner zu bedenken, dass es zufolge bestimmter Umstände, wie die Lage des $\mathrm{Ob}$ jektes, die sozialen Verhältnisse der Benütze des abgedichteten Bauwerkes, usw., ratsam des abgedichteten Bauwerkes, usw., ratsam die von der Vorschrift festgelegte minimale zu wählen.

Schliesslich wird noch ein Verfahren für den rechnungsmässigen Vergleich des Alterungs und Ablösungsfestigkeitskoeffizienten des Asphaltkittes bei verschiedenen Schrumpf dichtungsarten angegeben. 


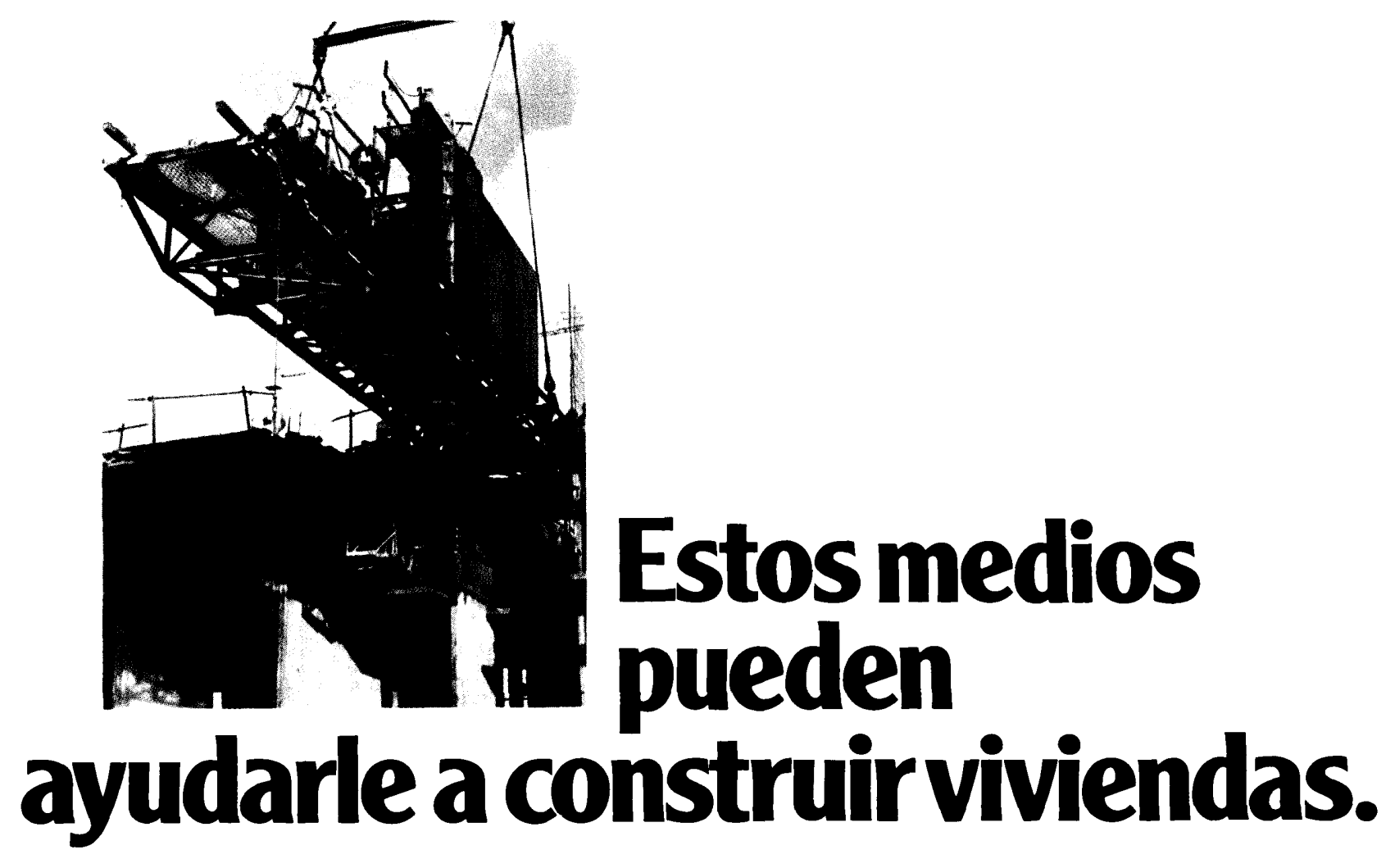

Dragados y Construcciones ha utilizado eficazmente sus medios para la realización de obras públicas.

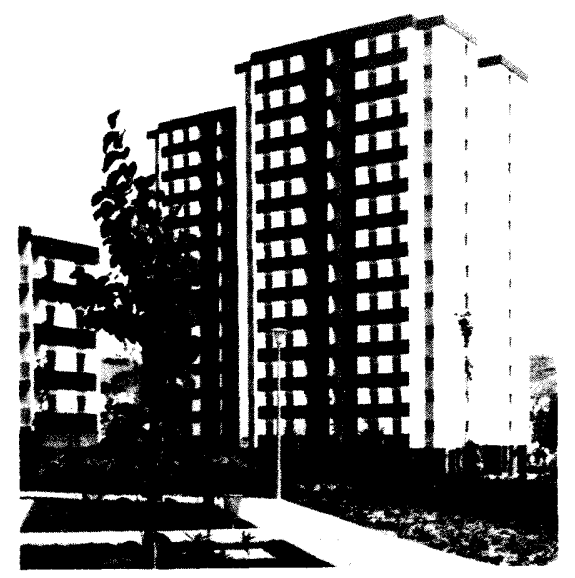

Lo que normalmente no se ha dicho, es que estos medios han contribuido también a la construcción de 200.000 viviendas.

Viviendas que equivalen a albergar la población de una ciudad de un millón de habitantes.

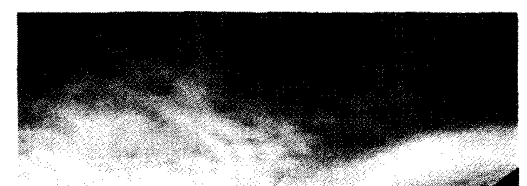

Ponemos al servicio del profesional de la edificación, nuestros hombres, nuestra experiencia y nuestros medios.

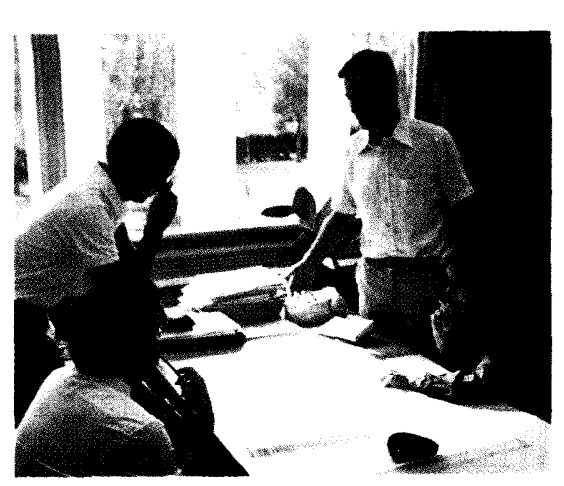

Las viviendas realizadas por Dragados y Construcciones, están dotadas con la infraestructura adecuada para todos y cada uno de los servicios comunitarios, inherentes a cualquier tipo de habitat.
Cuando promueva o proyecte viviendas, piense en Dragados y Construcciones, porque una buena idea ha de ser bien realizada.

\section{ORAGADOS Y CONSTRUCEIONES,S.A. \\ EMPRESA CONSTRUCTORA Domicilio Social \\ P. ${ }^{\circ}$ de la Alameda de Osuna, 50. Madrid-22 \\ DELEGACIONES EN TODO EL PAIS}




\section{2. definición del coeficiente de seguridad}

El valor $C$ del coeficiente de seguridad de un elemento constructivo se define, normalmente, como la relación entre su resistencia a la rotura $R$ y el esfuerzo máximo $F$ al que éste puede estar sometido en las circunstancias más desfavorables posibles.

Si tenemos, por ejemplo, una $R=3.600$ $\mathrm{kp} / \mathrm{cm}^{2}$ y un $F=1.200 \mathrm{kp} / \mathrm{cm}^{2}$ obtenemos como coeficiente de seguridad el valor de

$$
C=\frac{3.600}{1.200}=3,0
$$

Teniendo la absoluta seguridad de que en circunstancias reales el valor de $R_{1}$ de la resistencia real a la rotura no puede ser inferior a $R, R_{1} \geqslant R$, y que el valor de $F_{1}$ del esfuerzo máximo real no puede ser superior a $F, F_{1} \leqslant F$, podemos conformarnos con un mínimo valor del coeficiente de seguridad $C_{1}$, o sea, muy poco superior al valor de uno, fijando el valor admisible de $F_{1}$ algo inferior al valor de $F$.

Fijar el valor adecuado del coeficiente de seguridad es un asunto de gran responsabilidad. La industria aeronáutica, por ejemplo, es sin duda una de las más avanzadas en lo referente a la investigación, control de la ejecución y normas rigurosas del mantenimiento. Sin embargo, tenemos todos en la memoria la gran conmoción que sufrió el mundo civilizado al enterarse de que en EE.UU. de América un avión DC-10 se estrelló en circunstancias normales de vuelo debido a la rotura de uno de los tornillos que sujetaban un motor de propulsión. La causa de este fallo se atribuyó a la fatiga del material, lo que llama la atención sobre la importancia del factor tiempo en la apreciación del coeficiente de seguridad.

Naturalmente, no vamos a equiparar la trascendencia del coeficiente de seguridad en la industria aeronáutica con el de la impermeabilización de obras, pero nos parece importante tener el hecho presente, en la medida correspondiente, al pasar a ocuparnos de este último.

\section{3. definición del coeficiente de seguridad en las obras de impermeabilización}

El coeficiente de seguridad está definido en el apartado anterior en forma numérica basada en un cálculo, y expresa la relación entre la resistencia y el esfuerzo a que está sometido un elemento constructivo determinado, siendo condición indispensable el conocimiento de estos dos factores.

Tratándose de obras de impermeabilización la situación es, desgraciadamente, bien distinta. Tenemos conocimiento de los estudios que se están realizando desde hace varios años, lo mismo en Alemania que en Francia, con el fin de poder hacer un proyecto de impermeabilización con materiales bituminosos, basándose en la resistencia y el comportamiento de éstos y en los esfuerzos a que están sometidos.

En un trabajo colectivo de Braun-MetelmannThun-Vordermeier, publicado en el año 1976, se expone el procedimiento para el cálculo de impermeabilizaciones bituminosas (1). Con ocasión de las Jornadas Técnicas Internacionales de A.I.E., celebradas en Venecia en el año 1977, el Dr. Braun pronunció una conferencia sobre el mismo tema (2).

En otra conferencia, durante las mismas Jornadas Técnicas, J. Y. Meynard expuso una teoría del trabajo elástico del revestimiento impermeable de una cubierta, dando a conocer los resultados de los ensayos realizados en un laboratorio para determinar la anchura admisible de una grieta en el soporte base (3). 
Indudablemente se han conseguido resultados muy notables y prometedores para establecer una base científica para el proyecto de una impermeabilización bituminosa, pero estos procedimientos no se han generalizado aún. Las Normas vigentes se basan en las características mínimas exigibles de los materiales y de las membranas impermeabilizantes, cuya eficacia haya sido ampliamente comprobada por la experiencia en circunstancias determinadas.

Ante esta situación no podemos, naturalmente, obtener un coeficiente de seguridad en forma numérica, basado en un cálculo, teniendo que conformarnos con un coeficiente de seguridad relativo o comparativo, o sea, comparando, para un caso determinado, la eficacia de distintas soluciones.

Pero es indudable que, en las mismas condiciones de la obra, al aumentar por ejemplo el número de láminas de una membrana impermeabilizante, aumentamos también su seguridad y viceversa.

Unicamente y tratándose de juntas de dilatación, tenemos conocimiento de la posibilidad de comparar, por medio del cálculo, el coeficiente de resistencia al envejecimiento y al desprendimiento de la masilla asfáltica de sellado de distintos tipos de juntas. Volveremos sobre el particular en los apartados 14.1 y 14.2 .

\section{4. coeficiente de seguridad comparativo}

\begin{abstract}
Una demostración clara del significado y de la aplicación del coeficiente de seguridad comparativo nos ofrece, por ejemplo, la Norma francesa al exponer la incidencia de la pendiente sobre el tipo de la membrana impermeabilizante (4).
\end{abstract}

Se sabe que entre dos cubiertas de idénticas características, con la única diferencia de la pendiente, la de mayor pendiente ofrece una mayor seguridad contra las filtraciones, ya que favorece la evacuación de agua. La Norma refleja esta circunstancia rebajando o aumentando, en la composición de la membrana impermeabilizante, el número o la calidad de las láminas impermeabilizantes de acuerdo con la pendiente. Citamos a continuación los siguientes ejemplos:

Para una pendiente entre el 1 y el $5 \%$ se exigen una lámina tipo 18 y otra tipo 40; en cambio, para una pendiente del $0 \%$ hay que emplear dos láminas del tipo 18 y una del tipo 40 .

Para una pendiente entre el 5 y el $15 \%$ y un tipo de membrana autoprotegida, hay que emplear dos láminas tipo 40; en cambio, para una pendiente superior al $15 \%$, se debe emplear una del tipo 18 y otra del tipo 40 .

Otro ejemplo de la aplicación del coeficiente de seguridad comparativo nos ofrece la Norma alemana referente a la impermeabilización en presencia de agua a presión, aumentando el número de láminas impermeabilizantes de acuerdo con la presión del agua. (DIN 4.031 Impermeabilización en presencia de agua a presión).

Uno de los objetivos fundamentales de la Norma se manifiesta con toda claridad en los citados ejemplos. Partiendo de soluciones mínimas exigibles, de eficacia comprobada, hay que reforzarlas, o sea, aumentar su coeficiente de seguridad cuando las circunstancias de la obra lo hacen aconsejable.

\section{5. observaciones preliminares}

Antes de proceder a exponer los factores que condicionan el coeficiente de seguridad en las obras de impermeabilización, nos parece conveniente hacer constar las siguientes observaciones:

La impermeabilización no es un elemento constructivo independiente, no es un paraguas, sino que forma parte integrante del conjunto de la obra, dependiendo la eficacia de la impermeabilización, de su protección, del soporte base y, en general, de las características de la obra (5).

Al mismo tiempo existe una diferencia fundamental entre la obra de impermeabilización y la obra de albañilería. Mientras alguna pequeña deficiencia en la albañilería, normalmente, no tiene gran importancia, un peque- 
ño defecto en la impermeabilización puede ser la causa para la inutilización de un depósito de agua, o hacer inservible un local, ya que el agua acusa cualquier fallo. Por esta razón es preciso prestar una especial atención al coeficiente de seguridad en las obras de impermeabilización, teniendo presente que los tipos de impermeabilización que figuran en la Norma son siempre soluciones mínimas admisibles.

\section{6. circunstancias que condicionan el coeficiente de seguridad en una obra de impermeabilización}

Estas circunstancias podemos clasificarlas en la forma siguiente:

a) Las directamente relacionadas con la propia impermeabilización.

b) Las relacionadas con las características de la obra a impermeabilizar.

c) Emplazamiento de la obra.

d) Existencia de un seguro que garantice la eficacia de la impermeabilización.

e) Condición social de los usuarios de la obra impermeabilizada y el uso y mantenimiento de la misma.

A continuación nos ocuparemos detalladamente de estas circunstancias.

\section{7. circunstancias relacionadas directamente con la propia impermeabilización}

Con el fin de no extendernos demasiado, vamos a centrarnos sobre la impermeabilización de superfice por medio de una membrana impermeabilizante, por representar ésta uno de los trabajos de impermeabilización más frecuentes, pero siendo válidas también las conclusiones que se sacan para otras clases de impermeabilización, con las restricciones naturales.
No nos vamos a ocupar de la impermeabilización integral de la masa del hormigón o del mortero por medio de un aditivo impermeabilizante, ya que tiene características particulares, y hacemos referencia a la publicación del autor "Consideraciones sobre el Hormigón Impermeable y el Aspecto de su Garantía» (6).

Suponiendo que el tipo previsto de la membrana impermeabilizante es el adecuado, la eficacia de la impermeabilización depende de los cuatro siguientes factores:

1) Debida preparación del soporte base $y$ sus características.

2) Calidad de los materiales.

3) Calidad de la ejecución.

4) Protección de la impermeabilización.

\section{1. soporte base}

Tratándose de hormigón como soporte base, su acabado, a pesar de ser de fácil comprobación, resulta deficiente en muchas ocasiones, sea debido a una rugosidad excesiva, sea por permitir un embalsamiento de agua en algunos sitios, etc., viéndose obligada a transigir la empresa impermeabilizadora, para no enfrentarse con su cliente, la empresa constructora.

La falta de la resistencia mecánica del soporte base es difícilmente detectable por el impermeabilizador y no debe ser, lógicamente, imputable a éste una responsabilidad al observarse alguna filtración debida a la citada falta, pero, no obstante, le causa, por lo menos, una situación embarazosa.

Las características de las distintas clases del soporte base tienen mucha influencia sobre la eficacia de la impermeabilización, y este hecho fue ampliamente expuesto en una conferencia de P. Geisen con ocasión de las citadas Jornadas Técnicas de A.I.E., en Venecia (7).

Dada la importancia de este problema, en otra conferencia trató $M$. Stam de la evolución y problemática de la aplicación de la impermeabilización bituminosa sobre distintas clases de aislamiento térmico, haciendo especial referencia sobre la utilización de ciertos productos sintéticos como aislamiento térmico, y aprovechándolos como soporte base, lo que dio lugar a numerosas reclamaciones (8). 


\section{2. calidad de los materiales}

La empresa impermeabilizadora ha de confiar que los materiales que lleguen a pie de obra son de buena calidad, y que cumplan las exigencias de la Norma, ya que, generalmente, no se dispone de los medios necesarios para realizar la comprobación debida, salvo una revisión ocular. Solamente en obras excepcionales se realizan ciertos ensayos que se consideran más significativos.

\section{3. calidad de la ejecución}

Es decisivo para la eficacia de la impermeabilización la calidad de la ejecución, o sea, el reparto uniforme de los materiales, su colocación, el solape y la unión de las distintas piezas, el tratamiento adecuado de los puntos singulares, etc.

En una obra con pocos operarios, lo que ocurre muy frecuentemente, no hay posibilidad de disponer de una vigilancia técnica constante $y$ hay que confiar en la seriedad y capacidad profesional del operario colocador.

\section{4. protección de la impermeabilización}

La membrana impermeabilizante es sumamente vulnerable durante el proceso de la ejecución y antes de ser debidamente protegida al estar expuesta a un deterioro causado por un tráfico indebido $u$ otras incidencias propias de una obra en ejecución.

\section{8. circunstancias relacionadas con las características de la obra a impermeabilizar}

Se presupone que en el proyecto de la impermeabilización fueron tomadas en cuenta las características de la obra, como por ejemplo, las condiciones climatológicas, la presión de agua, etc. Ya nos hemos adelantado en lo referente a la repercusión de la pendiente sobre el tipo de la membrana impermeabilizante, y también nos hemos ocupado ya de la importancia del soporte base (ver apartados 4 y 7.1 ).
El no prestar la debida atención a ciertos detalles constructivos de la cubierta plana puede provocar la aparición de grietas en la misma y en las paredes de la última planta, afectando a su impermeabilidad, de acuerdo con lo expuesto por L. Logeais en su extenso y bien documentado artículo publicado en la Revue Générale de l'Etenchéité (9).

Como director de la Cía. de Seguros Bureau Sécuritas, L. Logeais pudo examinar unos tres mil expedientes de siniestros de su compañia y de la Cía. de Seguros Socotec, resultando que casi dos tercios eran debidos a algún defecto de la obra de hormigón armado en la última planta.

Para la fijación del coeficiente de seguridad adecuado hay que tener presente también las condiciones de trabajo y las consecuencias de algún defecto de la impermeabilización.

\section{1. condiciones de trabajo}

En los sitios donde las condiciones de trabajo son especialmente difíciles, o cuyo acceso requiere la instalación de costosos medios auxiliares, lo que haría complicada una eventual reparación, es recomendable reforzar la impermeabilización.

\section{2. consecuencias de algún defecto de la impermeabilización}

También se impone el refuerzo de la impermeabilización si se aprecian como muy graves los posibles daños que puede causar un defecto en la misma, lo que puede ocurrir en una sala de espectáculos instalada en un subterráneo, en unas instalaciones delicadas, o cuando se trata de objetos de un gran valor artístico, etc., y hacemos referencia a la publicación del autor «La Garantía en las Obras de Impermeabilización» (10).

\section{9. emplazamiento de la obra}

Para la apreciación del coeficiente de seguridad recomendable hay que tener en cuenta también el emplazamiento de la obra. Una obra de fácil acceso situada en un lugar céntrico se vigila, normalmente, con mayor frecuencia que una obra apartada y de difícil acceso. 
En este último caso, el resultado de la impermeabilización depende en un mayor grado de la pericia y de la seriedad profesional del operario colocador, por lo que resulta recomendable compensar la deficiencia de la vigilancia aumentando el coeficiente de seguridad.

\section{1. averías}

En una edificación apartada y aislada, habitada solamente durante ciertas temporadas, concurre además otra circunstancia desfavorable que conviene tener presente.

Al ocurrir alguna avería en la impermeabilización, transcurre muchas veces un tiempo considerable hasta que el interesado queda enterado del hecho, pudiendo producirse mientras tanto daños de consideración.

El conseguir que se realice la reparación necesaria presenta también, casi siempre, serias dificultades, dado el emplazamiento de la obra.

Por cierto, nos encontramos también con alguna frecuencia con el mismo problema en los edificios destinados a escuelas y que quedan deshabitados durante la época de vacaciones.

\section{0. seguro de la impermeabilización}

El hecho de estar la obra acogida a un seguro que garantice la eficacia de la impermeabilización repercute favorablemente sobre el coeficiente de seguridad, ya que presupone la conformidad de la compañía aseguradora con el proyecto de la impermeabilización y comprende la supervisión de su ejecución y la recepción de la obra acabada.

Significa además que el interesado puede contar con un responsable de solvencia para hacer frente a una reclamación justificada.

También, el mero hecho de disponer de una recepción de la obra por personal competente, disminuye notablemente el riesgo de algún fallo.

\section{1. condición social de los usuarios de la obra impermeabilizada, y el uso y mantenimiento de la misma}

Para poder cumplir con su cometido, el uso de la obra impermeabilizada debe ser el previsto, sus componentes debidamente mantenídos, los desperfectos subsanados, la evacuación de agua no obstaculizada y los desagües no obstruidos, criterio éste expuesto, por ejemplo, en las Normas Tecnológicas (11).

Tratándose de usuarios pertenecientes a una clase social económicamente desahogada, al observarse algún defecto, se procura, normalmente, remediarlo lo más rápidamente posible, antes de que se produzcan mayores daños y molestias.

Lamentablemente, éste no es el caso cuando los usuarios pertenecen a una clase social de modestos medios económicos. Ante la falta de recursos, se ven muchas veces imposibilitados de conseguir en un tiempo prudencial las reparaciones necesarias, agravándose mientras tanto los daños y molestias.

Por consiguiente, resulta aconsejable aumentar el coeficiente de seguridad de la impermeabilización, destinada a la vivienda modesta.

\section{2. evolución de la membrana impermeabilizante}

Al analizar los procedimientos que se emplean para componer una membrana impermeabilizante, se observa, en los últimos años, una tendencia a reducir el número de componentes del llamado sistema "multicapa» que figura en las distintas Normas, llegando hasta proponer membranas impermeabilizantes a base de una sola capa, o sea, la «monocapa». Ante este hecho nos parece importante hacer una breve exposición recordato- 
ria de la evolución de la membrana impermeabilizante.

Sabemos que en los comienzos de la técnica moderna de la impermeabilización al presentarse la necesidad de impermeabilizar, por ejemplo, una cubierta plana, se recurría al procedimiento más sencillo y económico posible, consistiendo en muchas ocasiones en la simple aplicación de dos pasadas de una pintura asfáltica.

Al observar los fallos de esta clase de impermeabilización, se aumentaba el número de las capas y se empleaban materiales más idóneos, hasta llegar al procedimiento de multicapa.

El principio fundamental que caracteriza esta solución puede expresarse de la manera siguiente:

a) Disponer de un espesor suficiente para obtener la necesaria resistencia mecánica y contra el envejecimiento.

b) Al contar con la posibilidad de algún fallo, lo mismo en el material que en la colocación, poder confiar que éstos no coincidan en el mismo sitio.

c) Simplificar en lo posible la ejecución de los solapes, punto clave para la eficacia de la impermeabilización.

Un inconveniente de la membrana impermeabilizante multicapa es el gran número de sus componentes $y$, por consiguiente, el elevado coste de la mano de obra.

Con el propósito de reducir el número de capas se procedió, por un lado, a aumentar el espesor de las láminas impermeabilizantes, y, por otro lado, a emplear materiales nuevos, en busca de la mejora de la calidad de la impermeabilización.

Ante la tendencia de una reducción excesiva del número de los componentes de la membrana impermeabilizante, es importante llamar la atención sobre el peligro que existe, al no respetar los limites que nos marca la experiencia y que llevaron precisamente a la creación de la membrana multicapa.

Observamos que, en algunas ocasiones, haciendo caso omiso de la necesidad de un espesor suficiente, que proporcione la resistencia mecánica necesaria, se dan a conocer por parte de la Industria Química soluciones para la impermeabilización de cubiertas, por ejemplo, basadas en la aplicación de dos pa- sadas excesivamente delgadas de cierto producto químico sintético. No dudamos de las cualidades excelentes del mismo, pero hay que tener presente que una obra no es un laboratorio, ni tampoco un taller. Efectivamente, después de algún tiempo no se vuelve a hablar más de aquella solución.

Una lámina gruesa sí que tiene la resistencia mecánica suficiente, pero, al exagerar su espesor, se dificulta su manejo y nos encontramos con serios problemas para su debida unión, sobre todo en los sitios donde coinciden más de dos piezas.

En lo que se refiere al modo de empleo de materiales nuevos, hay que señalar la seria divergencia que existe entre los fabricantes y los colocadores. En busca de competitividad, sobre todo para poder competir con los productos tradicionales, el fabricante procura reducir al máximo los espesores y las anchuras de los solapes y, por supuesto, el número de los componentes de la membrana impermeabilizante, llegando a considerar como suficiente el empleo de una sola lámina, o sea, una membrana monocapa, basándose en la calidad de sus productos.

El colocador, por el contrario, consciente de su responsabilidad y conocedor de las condiciones reales de la obra, aspira a poder contar con un coeficiente de seguridad lo más amplio posible. Le parece precario depender de la perfección de un solo elemento para garantizar la eficacia de una impermeabilización.

Al observarse algún defecto, se recurre siempre a la empresa colocadora, mientras que la empresa suministradora suele rechazar cualquier responsabilidad, atribuyendo el fallo a una colocación defectuosa. Una vez colocado, resulta muy dificil y costoso demostrar algún defecto del material, no siendo éste muy pronunciado, sobre todo al no coincidir la entrada de agua con la manifestación de la humedad, lo que ocurre, normalmente, al emplear un sistema no adherido de impermeabilización.

Vista esta situación se facilitaría, seguramente, la introducción de nuevos productos si los fabricantes participaran conjuntamente con los colocadores en la garantía de su eficacia. Pero, hasta la fecha, no tenemos ninguna noticia de esta clase de colaboración, limitándose los fabricantes a vender sus productos e inhibiéndose de sus resultados. 
Referente al empleo de la membrana impermeabilizante monocapa, y teniendo en cuenta lo expuesto anteriormente, nos parecen sumamente acertadas las aclaraciones que hizo el Dr. Braun en contestación a una pregunta, con ocasión de las Jornadas Técnicas de A.I.E. celebradas en Venecia en 1977 (12).

A pesar de ser también un fabricante, el Dr. Braun declara que no se encuentra en absoluto a gusto ante el riesgo y la garantía que supone el que la seguridad de la impermeabilización dependa de una sola capa. Está enterado, además, que lo mismo les ocurre a la mayoría de sus colegas de profesión en Alemania.

Prosigue manifestando el Dr. Braun que, aunque consciente de que el resultado de la impermeabilzación depende de la perfección de cada una de las uniones de las distintas piezas, no pueden ni él ni su encargado estar siempre presentes para controlar su correcta ejecución.

Sabemos que se dispone de procedimientos para localizar, por ejemplo, unos poros en una lámina impermeabilizante, y controlar la ejecución de la unión entre las distintas piezas, pero, contando con el factor humano y las circunstancias imprevisibles, nos adherimos por completo al criterio del Dr. Braun referente al empleo de una membrana monocapa.

En Francia, al estar generalizada la garantía de la construcción, incluidas las obras de impermeabilización, por medio de una póliza de seguro, son las compañías aseguradoras las que impiden, prácticamente y basándose en su experiencia, el empleo de aquellos procedimientos que no les ofrecen las debidas condiciones de seguridad, no admitiendo su seguro, por lo menos, en condiciones normales.

Ya que estas compañías de seguro son, en última instancia, las que tienen que hacer frente a alguna reclamación, debe considerarse, en casos dudosos, como muy valiosa su apreciación de la seguridad de un proyecto de impermeabilización.

\section{3. reparaciones}

El hecho de que una obra impermeabilizada necesite una reparación total, o también de una parte significativa de la misma, hay que interpretarlo como un toque de atención, sugiriendo la pregunta ¿por qué ha fallado la impermeabilización?

No hay que dejarse llevar a una explicación cómoda, atribuyendo el fallo a una deficiencia de la ejecución o de los materiales. Esto es naturalmente posible pero, tratándose de empresas acreditadas, resulta poco probable un fallo generalizado de esta clase.

Para enfocar debidamente una reparación, es de suma importancia aclarar primero la causa - las causas del fallo, ya que muchas veces suelen coincidir varios factores para provocarlo. Nos hemos ocupado detalladamente de las numerosas circunstancias que condicionan la eficacia de una impermeabilización, que pueden ser, aparte de las ya mencionadas, el tipo de la membrana impermeabilizante, deficiencia del soporte base, características de la estructura, etc.

Al persistir alguna duda sobre la causa del fallo y ante esta incógnita, es aconsejable aumentar el coeficiente de seguridad de la reparación. Esta medida resulta además recomendable ante la comprensible existencia de cierta desconfianza debida precisamente al fallo ocurrido con el fin de evitar, en lo posible, la necesidad de una nueva reparación.

\section{4. juntas de contracción en obras hidráulicas}

La resistencia al envejecimiento y al desprendimiento son dos de los distintos factores que condicionan la eficacia de una junta de contracción en obras hidráulicas, sellada por medio de una masilla asfáltica. Ambas clases de resistencia dependen, naturalmente, de las caracteristicas de la masilla pero, al mismo tiempo, también de las dimensiones de la junta. 
Partiendo de las caracteristicas análogas de la masilla de sellado, podemos establecer una relación entre las dimensiones de la junta y su resistencia al envejecimiento $y$ al desprendimiento. Una vez conocida esta relación para varios tipos de juntas, obtenemos su coeficiente de resistencia al envejecimiento $y$ al desprendimiento, lo que nos proporciona una medida para comparar su eficacia ante ambos fenómenos.

\section{1. coeficiente de resistencia al envejecimiento}

El envejecimiento es debido a la acción de agentes atmosféricos, por lo que el envejecimiento de la masilla asfáltica empieza en la zona de su contacto con aquéllos. Cuanto más extensa es esta zona, más intenso resulta el efecto del envejecimiento. Por otro lado, la superficie envejecida actúa como una pantalla de protección, atenuando la acción de los agentes atmosféricos; de esta forma, debajo de la capa endurecida, la masilla continúa en buen estado de conservación. Este estado de cosas depende, naturalmente, del espesor de la masilla y del tiempo de exposición al envejecimiento.

Por consiguiente, y partiendo de la misma calidad del producto, los dos factores que determinan la resistencia de la masilla al envejecimiento son: su superficie de contacto $S_{\text {c }}$ y su volumen $V$. La relación entre estos dos factores, volumen/superficie, o sea, $\mathrm{V} / \mathrm{S}$, representa el valor $\mathrm{R}$, del coeficiente de resistencia al envejecimiento de la masilla de sellado, de una junta de contracción determinada

$$
\mathrm{R}_{c}=\mathrm{V} / \mathrm{S}
$$

Al mayor valor de $R$, corresponde una mayor resistencia contra el envejecimiento $y$ viceversa.

Vamos a comparar a continuación el valor

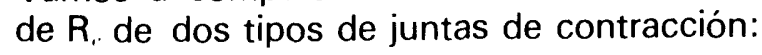

- Junta tipo n. ${ }^{\circ}$ 1, fig. 1, anchura $2 \mathrm{~cm}$, espesor de la masilla $10 \mathrm{~cm}$ :

$$
\mathrm{V}=20 ; \mathrm{S}_{1}=2 ; \mathrm{R}_{11}=20: 2=1 \mathrm{U}
$$

- Junta tipo $n .^{\circ} 2$, fig. 2 ( $\sin$ la capa de mortero de protección), anchuras 4 y $3 \mathrm{~cm}$ respectivamente $y$ espesor de la masilla $5 \mathrm{~cm}$ :

$$
\begin{aligned}
& V=\frac{4+3}{2} \times 5=17,5 \\
& S_{c}=4 \\
& R_{r, 2}=17,5: 4=4,4 \\
& R_{r 1}: R_{r 2}=10: 4,4=2,3 .
\end{aligned}
$$

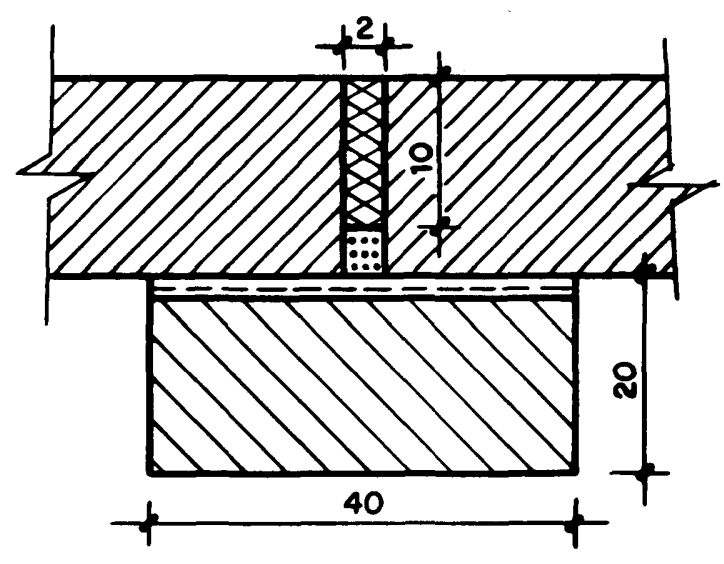

Fig. 1

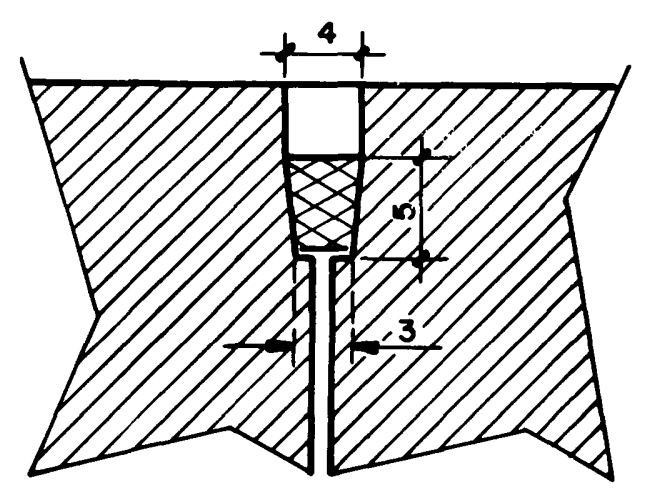

Fig. 2 
Es evidente que a pesar de emplear, prácticamente, el mismo volumen de masilla, la junta tipo $n .^{\circ} 1$ ofrece una resistencia al envejecimiento muy superior a la de la junta tipo n. ${ }^{\circ} 2$; por esta razón se precisa para esta última una capa de protección de mortero de acuerdo con la fig. 2.

\section{2. coeficiente de resistencia al desprendimiento}

Para que la junta cumpla con su cometido, es indispensable que la masilla de sellado, de consistencia pastora, se mantenga en su sitio, que no se produzca un vaciado parcial de la junta debido al escurrimiento o desprendimiento de la masilla.

Esta se mantiene en su sitio debido a su adherencia al perímetro de la junta, además de su propia resistencia al escurrimiento. A una mayor superficie adherida de la masilla, corresponde una mayor resistencia a su desprendimiento, actuando como contrapartida el peso propio de la misma. Contando con masillas con características análogas, y para una temperatura ambiente determinada, la relación entre la superficie adherida $S_{u}$ y el peso de la masilla, resulta decisiva para la apreciación de la resistencia al desprendimiento. Para mayor comodidad de cálculo, expresamos el peso de la masilla por su volumen $V$ y obtenemos la siguiente definición del valor $R_{d}$ del coeficiente de resistencia al desprendimiento.

$$
\mathrm{R}_{d}=\mathrm{S}_{a} / \mathrm{v}
$$

Al mayor valor de $R_{d}$, corresponde una mayor resistencia al desprendimiento, y viceversa.

Vamos a comparar a continuación el valor de $R_{d}$ de los mismos tipos de juntas, cuyos valores de $R$, hemos calculado anteriormente:

- Junta tipo n. ${ }^{\circ} 1$, fig. 1 :

$\mathrm{V}=20$

$\mathrm{S}_{a}=10+10+2=22$

$\mathrm{D}_{d 1}=22: 20=1,10$.

- Junta tipo n. ${ }^{\circ}$ 2, fig. 2 ( $\sin$ la capa de mortero de protección):

$V=17,5$

$\mathrm{S}_{a}=5+5+3=13$

$R_{c / 2}=13: 17,5=0,74$

$\mathbf{R}_{d 1}: \mathbf{R}_{d 2}=1,10: 0,74=1,5$.
La comparación de ambos coeficientes nos demuestra la notable superioridad de la resistencia al desprendimiento de la junta tipo $\mathrm{n} .^{\circ} 1$. Efectivamente, según lo demuestra la experiencia, al emplear la junta tipo n. ${ }^{\circ} 2$, hay que contar con la posibilidad del desprendimiento de la masilla de sellado, sobre todo con la temperatura ambiente elevada por lo que, normalmente, ésta se protege por medio de una capa de mortero de acuerdo con la fig. 2.

Interesa hacer constar que no es nuestra intención formar un criterio sobre la conveniencia del empleo de uno u otro tipo de junta, ya que esto depende también de otros factores no tratados en este trabajo; pretendemos, solamente en plan de ejemplo, establecer sus coeficientes de resistencia al envejecimiento y al desprendimiento.

\section{5. conclusión}

Es evidente que al depender la eficacia de una impermeabilización de un gran número de factores, tenemos que contar también con muchas posibilidades de fallo de alguno de éstos, por lo que hay que evitar un coeficiente de seguridad demasiado ajustado.

No se trata de una postura alarmista, sino de una postura realista, la que adopta, precisamente, la Norma al exigir las soluciones mínimas admisibles que ofrecen un coeficiente de seguridad adecuado de acuerdo con las circunstancias reales de la obra, o sea, comparando para un caso determinado la eficacia de distintas soluciones. Es indudable que, en las mismas condiciones de la obra, al aumentar, por ejemplo, el número de las capas de una membrana impermeabilizante, aumentamos también su coeficiente de seguridad y viceversa, criterio éste que se expresa en las Normas vigentes.

Hemos visto que son numerosas las circunstancias que influyen sobre la eficacia de la impermeabilzación, y por consiguiente condicionan su coeficiente de seguridad.

También hemos expuesto ciertas circunstancias especiales que hacen aconsejable buscar soluciones que ofrecen una seguridad superior a la mínima admitida por la Norma.

Esperamos, con este trabajo, haber aportado una contribución para aclarar el concepto de coeficiente de seguridad en las obras de impermeabilización. 